\title{
Harmonic Mean Inequalities for Hyperbolic Functions
}

\author{
Kwara Nantomah
}

\begin{abstract}
Department of Mathematics, Faculty of Mathematical Sciences,
C.K. Tedam University of Technology and Applied Sciences,

P. O. Box 24, Navrongo, Upper-East Region, Ghana

e-mail: knantomah@cktutas.edu.gh
\end{abstract}

\begin{abstract}
Inequalities involving hyperbolic functions have been the subject of intense discussion in recent times. In this work, we establish harmonic mean inequalities for these functions. This complements the results known in the literature. The techniques adopted in proving our results are analytical in nature.
\end{abstract}

\section{Introduction}

In 1974, Gautschi [9] established that for $z>0$, the harmonic mean of $\Gamma(z)$ and $\Gamma(1 / z)$ is always greater than or equal to 1 , where $\Gamma(z)$ is the classical gamma function. That is,

$$
\frac{2 \Gamma(z) \Gamma(1 / z)}{\Gamma(z)+\Gamma(1 / z)} \geq 1, \quad z>0 .
$$

Owing to the importance of this elegant inequality, some refinements and extensions have been studied. One may refer to [1, [2], [3], [4, [5], 6], 10] and [11] for such results.

In 2017, Alzer and Jameson [7] established that

$$
\frac{2 \psi(z) \psi(1 / z)}{\psi(z)+\psi(1 / z)} \geq-\gamma, \quad z>0
$$

where $\psi(z)$ is the digamma function.

Received: December 4, 2020; Revised \& Accepted: January 11, 2021

2010 Mathematics Subject Classification: 33B10, 33Bxx, 26D05.

Keywords and phrases: harmonic mean inequality, hyperbolic functions.

Copyright (c) 2021 the Author 
In 2018, Yin et al. [16] extended inequality (2) to the $k$-digamma function by proving that

$$
\frac{2 \psi_{k}(z) \psi_{k}(1 / z)}{\psi_{k}(z)+\psi_{k}(1 / z)} \geq \frac{\ln ^{2} k+\gamma^{2}-2(\gamma+1) \ln k}{k[\ln k+\psi(1 / k)]}, \quad z>0, \frac{1}{\sqrt[3]{3}} \leq k \leq 1 .
$$

In 2019, Nantomah [14] posed the inequality

$$
\frac{2 \beta(z) \beta(1 / z)}{\beta(z)+\beta(1 / z)} \leq \ln 2, \quad z>0
$$

as a conjecture, where $\beta(z)$ is the Nielsen's beta function [13]. Shortly thereafter, Matejicka [12] provided a concrete proof of (4).

Lately, Yildirim [15] improved on inequality (3) by relaxing the condition on $k$ and proving that

$$
\frac{2 \psi_{k}(z) \psi_{k}(1 / z)}{\psi_{k}(z)+\psi_{k}(1 / z)} \geq \psi_{k}(1), \quad z>0, k>0
$$

When $k=1$, inequalities (3) and (5) both reduce to inequality (2).

Also, Bouali [8] extended inequalities (1) and (2) to the q-gamma and $q$-digamma functions by proving that

$$
\frac{2 \Gamma_{q}(z) \Gamma_{q}(1 / z)}{\Gamma_{q}(z)+\Gamma_{q}(1 / z)} \geq 1, \quad z>0
$$

and

$$
\frac{2 \psi_{q}(z) \psi_{q}(1 / z)}{\psi_{q}(z)+\psi_{q}(1 / z)} \geq \psi_{q}(1), \quad z>0, q \in\left(0, p_{0}\right)
$$

where $p_{0} \simeq 3.239945$. By letting $q \rightarrow 1$, inequalities (6) and (7) respectively reduce to inequalities (1) and (2).

Inspired by the above works, the aim of this paper is to establish analogous results for the hyperbolic functions.

\section{Main Results}

The following lemmas help us to obtain a harmonic mean inequality for the hyperbolic sine function. 
Lemma 2.1. The inequality

$$
z \operatorname{coth}(z)>1
$$

holds for $z \in \mathbb{R} \backslash\{0\}$.

Proof. Let $g(z)=z \operatorname{coth}(z)$ for $z \in \mathbb{R} \backslash\{0\}$. Then

$$
g^{\prime}(z)=\operatorname{coth}(z)-z \operatorname{cosech}^{2}(z)=\frac{\operatorname{cosech}^{2}(z)}{2}[\sinh (2 z)-2 z] .
$$

Since $\sinh (z)>z$ for $z \in(0, \infty)$ and $\sinh (z)<z$ for $z \in(-\infty, 0)$, we conclude that the function $g(z)$ is increasing on $(0, \infty)$ and decreasing on $(-\infty, 0)$. Thus,

$$
g(z)>\lim _{z \rightarrow 0^{+}} g(z)=1
$$

for $z \in(0, \infty)$ and

$$
g(z)>\lim _{z \rightarrow 0^{-}} g(z)=1
$$

for $z \in(-\infty, 0)$. This completes the proof.

Lemma 2.2. The function $h(z)=z \operatorname{coth}(z) \operatorname{cosech}(z)$ is decreasing for $z \in \mathbb{R} \backslash\{0\}$. Proof. Let $z \in(0, \infty)$. Then by applying Lemma 2.1, we have

$$
h^{\prime}(z)=\operatorname{coth}(z) \operatorname{cosech}(z)[1-z \operatorname{coth}(z)]-z \operatorname{cosech}^{3}(z)<0 .
$$

By using the fact that the function $h^{\prime}(z)$ is even, we conclude that $h^{\prime}(z)<0$ for $z \in(-\infty, 0)$ as well. Hence $h(z)$ is decreasing for $z \in \mathbb{R} \backslash\{0\}$.

In the following theorem, we obtain some inequalities for the harmonic mean of $\sinh (z)$ and $\sinh (1 / z)$.

Theorem 2.3. The inequality

$$
0<\frac{2 \sinh (z) \sinh (1 / z)}{\sinh (z)+\sinh (1 / z)} \leq \frac{e^{2}-1}{2 e}
$$

holds for $z \in(0, \infty)$ and the inequality

$$
\frac{1-e^{2}}{2 e} \leq \frac{2 \sinh (z) \sinh (1 / z)}{\sinh (z)+\sinh (1 / z)}<0
$$

holds for $z \in(-\infty, 0)$. Equality holds in (8) and (9) if $z=1$ and $z=-1$ respectively. 
Proof. The cases for equality are obvious. So let $E(z)=\frac{2 \sinh (z) \sinh (1 / z)}{\sinh (z)+\sinh (1 / z)}$ for $z \in$ $\mathbb{R} \backslash\{0\}$ and let $\alpha(z)$ be defined as

$$
\begin{aligned}
\alpha(z) & =z[\operatorname{cosech}(z)+\operatorname{cosech}(1 / z)] \frac{E^{\prime}(z)}{E(z)} \\
& =z \operatorname{coth}(z) \operatorname{cosech}(z)-\frac{1}{z} \operatorname{coth}(1 / z) \operatorname{cosech}(1 / z) .
\end{aligned}
$$

Then by using Lemma 2.2, we get the following four cases.

(a) $\alpha(z)>0$ for $z \in(-\infty,-1)$,

(b) $\alpha(z)<0$ for $z \in(-1,0)$,

(c) $\alpha(z)>0$ for $z \in(0,1)$,

(d) $\alpha(z)<0$ for $z \in(1, \infty)$.

Since $\sinh (z)>0$ for $z \in(0, \infty)$ and $\sinh (z)<0$ for $z \in(-\infty, 0)$, we have $E(z)>$ 0 for $z \in(0, \infty), E(z)<0$ for $z \in(-\infty, 0)$ and $z[\operatorname{cosech}(z)+\operatorname{cosech}(1 / z)]>0$ for all $z \in \mathbb{R}$. Hence

(a) $\alpha(z)>0 \Rightarrow \frac{E^{\prime}(z)}{E(z)}>0 \Rightarrow E^{\prime}(z)<0$ for $z \in(-\infty,-1)$,

(b) $\alpha(z)<0 \Rightarrow \frac{E^{\prime}(z)}{E(z)}<0 \Rightarrow E^{\prime}(z)>0$ for $z \in(-1,0)$,

(c) $\alpha(z)>0 \Rightarrow \frac{E^{\prime}(z)}{E(z)}>0 \Rightarrow E^{\prime}(z)>0$ for $z \in(0,1)$,

(d) $\alpha(z)<0 \Rightarrow \frac{E^{\prime}(z)}{E(z)}<0 \Rightarrow E^{\prime}(z)<0$ for $z \in(1, \infty)$.

Thus, the function $E(z)$ is increasing on $(-1,0) \cup(0,1)$ and decreasing on $(-\infty,-1) \cup(1, \infty)$. Consequently, for $z \in(0,1)$, we have

$$
0=\lim _{z \rightarrow 0^{+}} E(z)<E(z)<\lim _{z \rightarrow 1^{-}} E(z)=\frac{e^{2}-1}{2 e}
$$

and for $z \in(1, \infty)$, we have

$$
0=\lim _{z \rightarrow \infty} E(z)<E(z)<\lim _{z \rightarrow 1^{+}} E(z)=\frac{e^{2}-1}{2 e} .
$$


Hence, inequality (8) holds. Also, for $z \in(-1,0)$, we have

$$
\frac{1-e^{2}}{2 e}=\lim _{z \rightarrow-1^{+}} E(z)<E(z)<\lim _{z \rightarrow 0^{-}} E(z)=0
$$

and for $z \in(-\infty,-1)$, we have

$$
\frac{1-e^{2}}{2 e}=\lim _{z \rightarrow-1^{-}} E(z)<E(z)<\lim _{z \rightarrow-\infty} E(z)=0 .
$$

Hence, inequality (9) holds. This completes the proof of the theorem.

The following lemma is required in order to prove our next results.

Lemma 2.4. The function $f(z)=z \operatorname{cosech}^{2}(z)$ is decreasing for $z \in \mathbb{R} \backslash\{0\}$.

Proof. Since $z \operatorname{coth}(z)>1$ for all $z \in \mathbb{R} \backslash\{0\}$, then direct computation yields

$$
f^{\prime}(z)=[1-2 z \operatorname{coth}(z)] \operatorname{cosech}^{2}(z)<0
$$

which completes the proof.

In the following theorem, we obtain some inequalities for the harmonic mean of $\tanh (z)$ and $\tanh (1 / z)$.

Theorem 2.5. The inequality

$$
0<\frac{2 \tanh (z) \tanh (1 / z)}{\tanh (z)+\tanh (1 / z)} \leq \frac{e^{2}-1}{e^{2}+1}
$$

holds for $z \in(0, \infty)$ and the inequality

$$
\frac{1-e^{2}}{e^{2}+1} \leq \frac{2 \tanh (z) \tanh (1 / z)}{\tanh (z)+\tanh (1 / z)}<0
$$

holds for $z \in(-\infty, 0)$. Equality holds in (10) and (11) if $z=1$ and $z=-1$ respectively. 
Proof. The cases for equality are obvious. So let $F(z)=\frac{2 \tanh (z) \tanh (1 / z)}{\tanh (z)+\tanh (1 / z)}$ for $z \in \mathbb{R} \backslash\{0\}$ and let $\beta(z)$ be defined as

$$
\begin{aligned}
\beta(z) & =z[\operatorname{coth}(z)+\operatorname{coth}(1 / z)] \frac{F^{\prime}(z)}{F(z)} \\
& =z \operatorname{cosech}^{2}(z)-\frac{1}{z} \operatorname{cosech}^{2}(1 / z) .
\end{aligned}
$$

Then by applying Lemma 2.4 , we obtain the following cases.

(i) $\beta(z)>0$ for $z \in(-\infty,-1)$,

(ii) $\beta(z)<0$ for $z \in(-1,0)$,

(iii) $\beta(z)>0$ for $z \in(0,1)$,

(iv) $\beta(z)<0$ for $z \in(1, \infty)$.

Since $\tanh (z)>0$ for $z \in(0, \infty)$ and $\tanh (z)<0$ for $z \in(-\infty, 0)$, we have $F(z)>0$ for $z \in(0, \infty), F(z)<0$ for $z \in(-\infty, 0)$ and $z[\operatorname{coth}(z)+\operatorname{coth}(1 / z)]>0$ for all $z \in \mathbb{R}$. Moreover,

(i) $\beta(z)>0 \Rightarrow \frac{F^{\prime}(z)}{F(z)}>0 \Rightarrow F^{\prime}(z)<0$ for $z \in(-\infty,-1)$,

(ii) $\beta(z)<0 \Rightarrow \frac{F^{\prime}(z)}{F(z)}<0 \Rightarrow F^{\prime}(z)>0$ for $z \in(-1,0)$,

(iii) $\beta(z)>0 \Rightarrow \frac{F^{\prime}(z)}{F(z)}>0 \Rightarrow F^{\prime}(z)>0$ for $z \in(0,1)$,

(iv) $\beta(z)<0 \Rightarrow \frac{F^{\prime}(z)}{F(z)}<0 \Rightarrow F^{\prime}(z)<0$ for $z \in(1, \infty)$.

Thus, the function $F(z)$ is increasing on $(-1,0) \cup(0,1)$ and decreasing on $(-\infty,-1) \cup(1, \infty)$. Because of this, for $z \in(0,1)$, we have

$$
0=\lim _{z \rightarrow 0^{+}} F(z)<F(z)<\lim _{z \rightarrow 1^{-}} F(z)=\frac{e^{2}-1}{e^{2}+1}
$$

and for $z \in(1, \infty)$, we have

$$
0=\lim _{z \rightarrow \infty} F(z)<F(z)<\lim _{z \rightarrow 1^{+}} F(z)=\frac{e^{2}-1}{e^{2}+1} .
$$


Hence, inequality 100 holds. Likewise, for $z \in(-1,0)$, we have

$$
\frac{1-e^{2}}{e^{2}+1}=\lim _{z \rightarrow-1^{+}} F(z)<F(z)<\lim _{z \rightarrow 0^{-}} F(z)=0
$$

and for $z \in(-\infty,-1)$, we have

$$
\frac{1-e^{2}}{e^{2}+1}=\lim _{z \rightarrow-1^{-}} F(z)<F(z)<\lim _{z \rightarrow-\infty} F(z)=0 .
$$

Hence, inequality (11) holds. This completes the proof of the theorem.

In the following theorem, we obtain some inequalities for the harmonic mean of $\cosh (z)$ and $\cosh (1 / z)$.

Theorem 2.6. The inequality

$$
\frac{e^{2}+1}{2 e}<\frac{2 \cosh (z) \cosh (1 / z)}{\cosh (z)+\cosh (1 / z)}<k
$$

holds for $z \in(0.161872635,1)$ or $z \in(1,6.177696420)$ and the inequality

$$
2<\frac{2 \cosh (z) \cosh (1 / z)}{\cosh (z)+\cosh (1 / z)}<k
$$

holds for $z \in(0,0.161872635)$ or $z \in(6.177696420, \infty)$. Where, $k=2.017775507$. Proof. Let $G(z)=\frac{2 \cosh (z) \cosh (1 / z)}{\cosh (z)+\cosh (1 / z)}$ and $\theta(z)=\ln G(z)$ for $z>0$ and $z \neq 1$. Then

$$
\theta^{\prime}(z)=\frac{\sinh (z)}{\cosh (z)}-\frac{1}{z^{2}} \frac{\sinh (1 / z)}{\cosh (1 / z)}-\frac{\sinh (z)-\frac{1}{z^{2}} \sinh (1 / z)}{\cosh (z)+\cosh (1 / z)}
$$

which implies that

$$
\begin{aligned}
z[\operatorname{cosech}(z)+\operatorname{cosech}(1 / z)] \theta^{\prime}(z) & =z \tanh (z) \operatorname{sech}(z)-\frac{1}{z} \tanh (1 / z) \operatorname{sech}(1 / z) \\
& =\gamma(z)
\end{aligned}
$$

The function $\gamma(z)$ has the roots $z_{1} \approx 0.161872635, z_{2}=1$ and $z_{3} \approx 6.177696420$. In addition, $\gamma(z)<0$ if $z \in\left(z_{1}, z_{2}\right)$ or $z \in\left(z_{3}, \infty\right)$ and $\gamma(z)>0$ if $z \in\left(0, z_{1}\right)$ or $z \in\left(z_{2}, z_{3}\right)$. Thus, $\theta^{\prime}(z)<0$ if $z \in\left(z_{1}, z_{2}\right)$ or $z \in\left(z_{3}, \infty\right)$ and $\theta^{\prime}(z)>0$ if 
$z \in\left(0, z_{1}\right)$ or $z \in\left(z_{2}, z_{3}\right)$. As a result of these, $G(z)$ is decreasing if $z \in\left(z_{1}, z_{2}\right)$ or $z \in\left(z_{3}, \infty\right)$ and increasing if $z \in\left(0, z_{1}\right)$ or $z \in\left(z_{2}, z_{3}\right)$. Then for $z \in\left(z_{1}, z_{2}\right)$ we have

$$
\frac{e^{2}+1}{2 e}=G\left(z_{2}\right)<G(z)<G\left(z_{1}\right)=2.017775507
$$

which gives the inequality $(12)$. Also, for $z \in\left(z_{2}, z_{3}\right)$ we have

$$
G\left(z_{2}\right)<G(z)<G\left(z_{3}\right)=2.017775507
$$

which coincides with 12$)$. Furthermore, for $z \in\left(0, z_{1}\right)$ we have

$$
2=\lim _{z \rightarrow 0^{+}} G(z)=G\left(0^{+}\right)<G(z)<G\left(z_{1}\right)
$$

which gives the inequality 13 . Likewise, for $z \in\left(z_{3}, \infty\right)$ we have

$$
2=\lim _{z \rightarrow \infty} G(z)=G(\infty)<G(z)<G\left(z_{3}\right)
$$

which agrees with (13). This completes the proof.

Remark 2.7. Since the function $G(z)$ in Theorem 2.6 is even, inequality 12 also holds for $z \in(-1,-0.161872635)$ or $z \in(-6.177696420,-1)$ and inequality (13) also holds for $z \in(-0.161872635,0)$ or $z \in(-\infty,-6.177696420)$.

In the following theorem, we obtain some inequalities for the harmonic mean of $\operatorname{cosech}(z)$ and $\operatorname{cosech}(1 / z)$.

Theorem 2.8. The inequality

$$
0<\frac{2 \operatorname{cosech}(z) \operatorname{cosech}(1 / z)}{\operatorname{cosech}(z)+\operatorname{cosech}(1 / z)} \leq \frac{2 e}{e^{2}-1}
$$

holds for $z \in(0, \infty)$ and the inequality

$$
\frac{2 e}{1-e^{2}} \leq \frac{2 \operatorname{cosech}(z) \operatorname{cosech}(1 / z)}{\operatorname{cosech}(z)+\operatorname{cosech}(1 / z)}<0
$$

holds for $z \in(-\infty, 0)$. Equality holds in (14) and 15) if $z=1$ and $z=-1$ respectively. 
Proof. The cases for equality are obvious. So let $H(z)=\frac{2 \operatorname{cosech}(z) \operatorname{cosech}(1 / z)}{\operatorname{cosech}(z)+\operatorname{cosech}(1 / z)}$ for $z \in \mathbb{R} \backslash\{0\}$. Then

$$
\begin{aligned}
z[\sinh (z)+\sinh (1 / z)] \frac{H^{\prime}(z)}{H(z)} & =\frac{1}{z} \cosh (1 / z)-z \cosh (z) \\
& =\phi(z) .
\end{aligned}
$$

Since the function $z \cosh (z)$ increasing for all real values, we conclude that $\phi(z)>$ 0 if $z \in(-\infty,-1)$ or $z \in(0,1)$ and $\phi(z)<0$ if $z \in(-1,0)$ or $z \in(1, \infty)$. These imply that $H(z)$ is increasing on $(-1,0) \cup(0,1)$ and decreasing on $(-\infty,-1) \cup$ $(1, \infty)$. Consequently, for $z \in(0,1)$, we have

$$
0=\lim _{z \rightarrow 0^{+}} H(z)<H(z)<\lim _{z \rightarrow 1^{-}} H(z)=\frac{2 e}{e^{2}-1}
$$

and for $z \in(1, \infty)$, we have

$$
0=\lim _{z \rightarrow \infty} H(z)<H(z)<\lim _{z \rightarrow 1^{+}} H(z)=\frac{2 e}{e^{2}-1} .
$$

Hence, inequality (14) holds. Also, for $z \in(-1,0)$, we have

$$
\frac{2 e}{1-e^{2}}=\lim _{z \rightarrow-1^{+}} H(z)<H(z)<\lim _{z \rightarrow 0^{-}} H(z)=0
$$

and for $z \in(-\infty,-1)$, we have

$$
\frac{2 e}{1-e^{2}}=\lim _{z \rightarrow-1^{-}} H(z)<H(z)<\lim _{z \rightarrow-\infty} H(z)=0 .
$$

Hence, inequality 15 holds. This completes the proof of the theorem.

In the following theorem, we obtain an inequality for the harmonic mean of $\operatorname{sech}(z)$ and $\operatorname{sech}(1 / z)$.

Theorem 2.9. The inequality

$$
0<\frac{2 \operatorname{sech}(z) \operatorname{sech}(1 / z)}{\operatorname{sech}(z)+\operatorname{sech}(1 / z)} \leq \frac{2 e}{e^{2}+1}
$$

holds for $z \in \mathbb{R} \backslash\{0\}$. Equality holds if $z=1$ or $z=-1$. 
Proof. First, we prove the results for $z \in(0, \infty)$. The case for $z=1$ is obvious. So, let $K(z)=\frac{2 \operatorname{sech}(z) \operatorname{sech}(1 / z)}{\operatorname{sech}(z)+\operatorname{sech}(1 / z)}$ for $z \in(0,1) \cup(1, \infty)$. Then

$$
z[\operatorname{sech}(z)+\operatorname{sech}(1 / z)] \frac{K^{\prime}(z)}{K(z)}=\frac{1}{z} \tanh (1 / z) \operatorname{sech}(z)-z \tanh (z) \operatorname{sech}(1 / z)
$$

which implies that

$$
\begin{aligned}
z[\cosh (z)+\cosh (1 / z)] \frac{K^{\prime}(z)}{K(z)} & =\frac{1}{z} \sinh (1 / z)-z \sinh (z) \\
& =\delta(z) .
\end{aligned}
$$

Since the function $z \sinh (z)$ increasing for all real values, we conclude that $\delta(z)>0$ if $z \in(0,1)$ and $\delta(z)<0$ if $z \in(1, \infty)$. Thus, $K(z)$ is increasing on $(0,1)$ and decreasing on $(1, \infty)$. Consequently, we obtain

$$
0=\lim _{z \rightarrow 0^{+}} K(z)<K(z)<\lim _{z \rightarrow 1^{-}} K(z)=\frac{2 e}{e^{2}+1}
$$

for $z \in(0,1)$ and

$$
0=\lim _{z \rightarrow \infty} K(z)<K(z)<\lim _{z \rightarrow 1^{+}} K(z)=\frac{2 e}{e^{2}+1}
$$

for $z \in(1, \infty)$. Hence the inequality (16) holds for all $z \in(0, \infty)$. Since $K(z)$ is an even function, it implies that (16) also holds for $z \in(-\infty, 0)$. This completes the proof of the theorem.

In the following theorem, we obtain some inequalities for the harmonic mean of $\operatorname{coth}(z)$ and $\operatorname{coth}(1 / z)$.

Theorem 2.10. The inequality

$$
\frac{e^{2}+1}{e^{2}-1} \leq \frac{2 \operatorname{coth}(z) \operatorname{coth}(1 / z)}{\operatorname{coth}(z)+\operatorname{coth}(1 / z)}<2
$$

holds for $z \in(0, \infty)$ and the inequality

$$
-2<\frac{2 \operatorname{coth}(z) \operatorname{coth}(1 / z)}{\operatorname{coth}(z)+\operatorname{coth}(1 / z)} \leq \frac{e^{2}+1}{1-e^{2}}
$$

holds for $z \in(-\infty, 0)$. Equality holds in (17) and (18) if $z=1$ and $z=-1$ respectively. 
Proof. The cases for equality are obvious. So let $T(z)=\frac{2 \operatorname{coth}(z) \operatorname{coth}(1 / z)}{\operatorname{coth}(z)+\operatorname{coth}(1 / z)}$ for $z \in$ $\mathbb{R} \backslash\{0\}$. Then

$$
\begin{aligned}
z[\tanh (z)+\tanh (1 / z)] \frac{T^{\prime}(z)}{T(z)} & =\frac{1}{z} \operatorname{sech}^{2}(1 / z)-z \operatorname{sech}^{2}(z) \\
& =\lambda(z) .
\end{aligned}
$$

It follows that $\lambda(z)>0$ if $z \in(-1,0)$ or $z \in(1, \infty)$ and $\lambda(z)<0$ if $z \in(-\infty,-1,0)$ or $z \in(0,1)$. Since $T(z)>0$ for $z \in(0, \infty)$ and $T(z)<0$ for $z \in(-\infty, 0)$, we conclude that $T(z)$ is increasing on $(-\infty,-1) \cup(1, \infty)$ and decreasing on $(-1,0) \cup(0,1)$. Consequently, for $z \in(0,1)$, we obtain

$$
\frac{e^{2}+1}{e^{2}-1}=\lim _{z \rightarrow 1^{-}} T(z)<T(z)<\lim _{z \rightarrow 0^{+}} H(z)=2
$$

and for $z \in(1, \infty)$, we obtain

$$
\frac{e^{2}+1}{e^{2}-1}=\lim _{z \rightarrow 1^{+}} T(z)<T(z)<\lim _{z \rightarrow \infty} H(z)=2 .
$$

Hence, inequality (17) holds. Also, for $z \in(-\infty,-1)$, we obtain

$$
-2=\lim _{z \rightarrow-\infty} T(z)<T(z)<\lim _{z \rightarrow-1^{-}} H(z)=\frac{e^{2}+1}{1-e^{2}}
$$

and for $z \in(-1,0)$, we obtain

$$
-2=\lim _{z \rightarrow 0^{-}} T(z)<T(z)<\lim _{z \rightarrow-1^{+}} H(z)=\frac{e^{2}+1}{1-e^{2}} .
$$

Hence, inequality (18) holds. This completes the proof of the theorem.

\section{Conclusion}

In this work, we have established harmonic mean inequalities for the hyperbolic functions. The inequalities provide lower and upper bounds for harmonic means of these functions. The results established could trigger further investigations on inequalities involving hyperbolic functions. Also, the techniques used could be adopted to establish similar results for trigonometric functions. 


\section{Acknowledgements}

The author would like thank the anonymous referees for the comments and suggestions which helped to improve the quality of this paper.

\section{References}

[1] H. Alzer, A harmonic mean inequality for the gamma function, J. Comp. Appl. Math. 87 (1997), 195-198. https://doi.org/10.1016/S0377-0427(96)00181-1

[2] H. Alzer, Inequalities for the gamma function, Proc. Amer. Math. Soc. 128 (1999), 141-147. https://doi.org/10.1090/S0002-9939-99-04993-X

[3] H. Alzer, On a gamma function inequality of Gautschi, Proc. Edinburgh Math. Soc. 45 (2002), 589-600. https://doi.org/10.1017/S0013091501000943

[4] H. Alzer, On Gautschi's harmonic mean inequality for the gamma function, J. Comp. Appl. Math. 157 (2003), 243-249.

https://doi.org/10.1016/S0377-0427(03)00456-4

[5] H. Alzer, Inequalities involving $\Gamma(x)$ and $\Gamma(1 / x)$, J. Comp. Appl. Math. 192 (2006), 460-480. https://doi.org/10.1016/j.cam.2005.04.063

[6] H. Alzer, Gamma function inequalities, Numer. Algor. 49 (2008), 53-84. https://doi.org/10.1007/s11075-008-9160-4

[7] H. Alzer and G. Jameson, A harmonic mean inequality for the digamma function and related results, Rend. Sem. Mat. Univ. Padova. 137 (2017), 203-209. https://doi.org/10.4171/RSMUP/137-10

[8] M. Bouali, A harmonic mean inequality for the $q$-gamma and $q$-digamma functions, arXiv:2005.08945 [math.CA].

[9] W. Gautschi, A harmonic mean inequality for the gamma function, SIAM J. Math. Anal. 5 (1974), 278-281. https://doi.org/10.1137/0505030

[10] C. Giordano and A. Laforgia, Inequalities and monotonicity properties for the gamma function, J. Comp. Appl. Math. 133 (2001), 387-396.

https://doi.org/10.1016/S0377-0427(00)00659-2 
[11] G. J. O. Jameson and T. P. Jameson, An inequality for the gamma function conjectured by D. Kershaw, J. Math. Ineq. 6 (2012), 175-181. https://doi.org/10.7153/jmi-06-18

[12] L. Matejicka, Proof of a conjecture On Nielsen's $\beta$-function, Probl. Anal. Issues Anal. 8(26) (2019), 105-111. https://doi.org/10.15393/j3.art.2019.6810

[13] K. Nantomah, On some properties and inequalities for the Nielsen's $\beta$-function, Sci. Ser. A Math. Sci. (N.S.) 28 (2017-2018), 43-54.

[14] K. Nantomah, Certain properties of the Nielsen's $\beta$-function, Bull. Int. Math. Virtual Inst. 9 (2019), 263-269.

[15] E. Yildirim, Monotonicity properties on $k$-digamma function and its related inequalities, J. Math. Inequal. 14(1) (2020), 161-173. https://doi.org/10.7153/jmi-2020-14-12

[16] L. Yin, L-G. Huang, X-L. Lin and Y-L. Wang, Monotonicity, concavity, and inequalities related to the generalized digamma function, Adv. Difference Equ. (2018) 2018:246. https://doi.org/10.1186/s13662-018-1695-7

This is an open access article distributed under the terms of the Creative Commons Attribution License (http://creativecommons.org/licenses/by/4.0/), which permits unrestricted, use, distribution and reproduction in any medium, or format for any purpose, even commercially provided the work is properly cited. 